\title{
Correlation of c-Axis Orientation of $\alpha$-Titanium Grains with Polarized Light Optical Microscopy Intensity Profiles
}

\author{
Ke-Wei Jin ${ }^{1}$, Marc De Graef ${ }^{1}$ \\ ${ }^{1}$ Dept. of Materials Science and Engineering, Carnegie Mellon Univ., Pittsburgh PA 15213, USA
}

In the study of materials science and engineering, the structure and symmetry of a material play an important role in the resulting properties and performance; in addition, the distribution of crystallographic orientations in a poly-crystalline material strongly affects the properties. While many engineering materials have high crystallographic symmetry, the increasing interest in multi-functional materials has led to the development and use of anisotropic, lower symmetry structures, which may have unique functional properties. Optical microscopy has long been used to study the microstructure of materials. When polarized light is incident on certain metals, such as $\alpha$-titanium, optically anisotropic effects have been observed [1]. Compared to other orientation determination methods such as scanning and transmission electron microscopy, optical microscopy is lower cost and has the ability to accommodate larger samples. In addition, optical microscopy can also capture other important information about microstructure such as size, shape, and distribution of grains.

Due to desirable properties such as high strength and corrosion resistance, titanium and its alloys are of interest in many engineering industries. The $c$-axis orientation of $\alpha$-titanium is of interest because the orientation of the hexagonal structure with respect to a mechanical loading direction can have consequences on the deformation behavior of titanium [2]. Additionally, the hexagonal structure of $\alpha$ titanium also results in anisotropic optical behavior. When illuminated under polarized light, the individual grains of a poly-crystalline $\alpha$-titanium sample can be identified readily.

Illuminated $\alpha$-titanium grains exhibit different reflected intensities, depending on the grain orientation (Fig. 1). In this method of orientation determination, the inclination and azimuth of the $c$-axis are of interest. As the orientation of the incident polarized light beam is changed with respect to the sample, the recorded intensity of the grains changes. Micrographs of an equiaxed $\alpha$-titanium sample were captured as the incident polarized light beam was rotated $360^{\circ}$ at $10^{\circ}$ increments. From the series of micrographs, the full intensity profile of each grain can be extracted.

The intensity profile of each grain is extracted from the polarized light microscopy data, and the $c$-axis orientation from electron back-scatter diffraction (EBSD). A plot of the maximum intensity value vs. the $c$-axis inclination shows an imperfect correlation (Fig. 2). Similarly, a correlation can be determined between the rotation index of the maximum intensity and the azimuthal angle, which shows that a $180^{\circ}$ degeneracy exists for the current experimental methods.

A forward model for the polarized light microscopy technique for orientation determination was created with the goal of performing dictionary indexing of the recorded polarized light intensity profiles. Forward models use a physics-based simulation to predict the result of an experiment, and have been used successfully in generating EBSD patterns [3]. For polarized light microscopy, the forward model was generated by using Mueller calculus to determine the result of light interaction with different optical elements and with the titanium sample. Mueller matrices for each titanium grain were calculated as a 
function of the orientation of the grain. We will report on a comparison between the forward model intensity profiles and the experimental polarized light intensity profiles.

\section{References:}

[1] GKT Conn and FJ Bradshaw in "Polarized Light in Metallography", (Buttersworth Scientific, London).

[2] A Zarkades and FR Larson, The Science, Technology and Application of Titanium (1970) p. 933941.

[3] K Marquardt et al, American Mineralogist 102 (2018) p.1843-1855

[4] The authors acknowledge ONR Vannevar Bush Faculty Fellowship support (N00014-16-1-2821), the Wright Patterson Air Force Research Laboratory as well as the computational resources of the Materials Characterization Facility at CMU, grant MCF-677785.
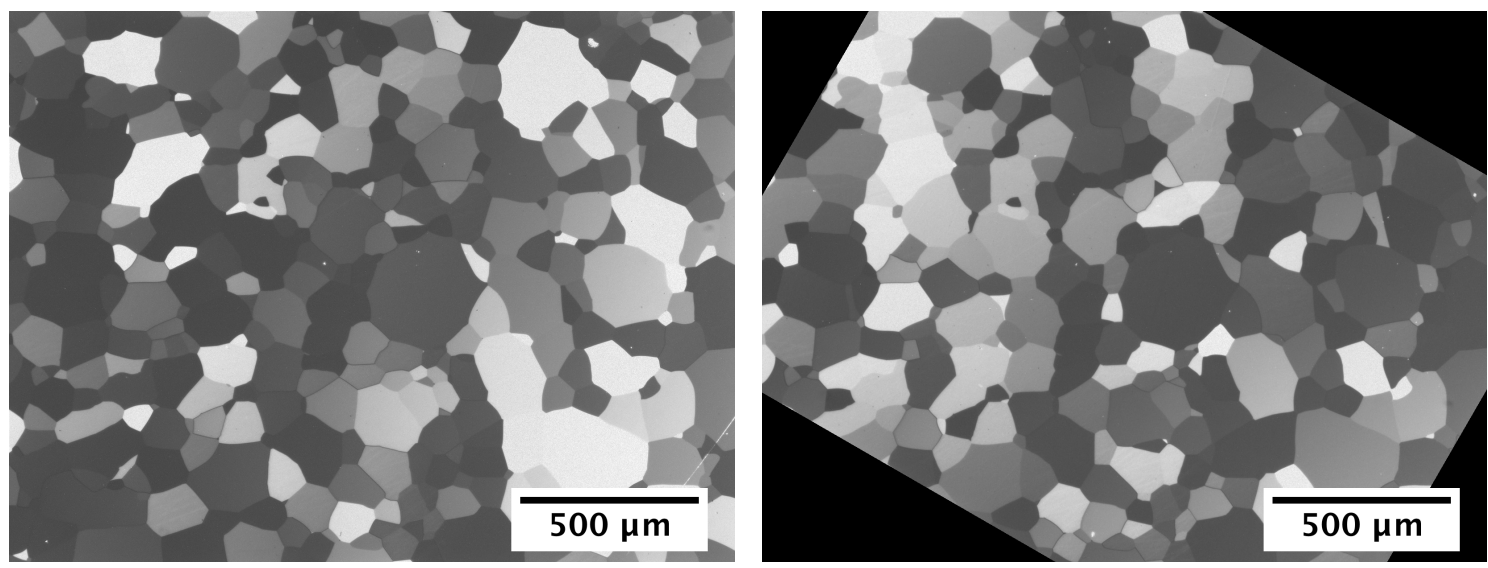

Figure 1. Polarized light micrographs of an $\alpha$-Ti sample at $0^{\circ}$ (left) and at $30^{\circ}$ (right). At different angles, the grains have different reflected intensities.
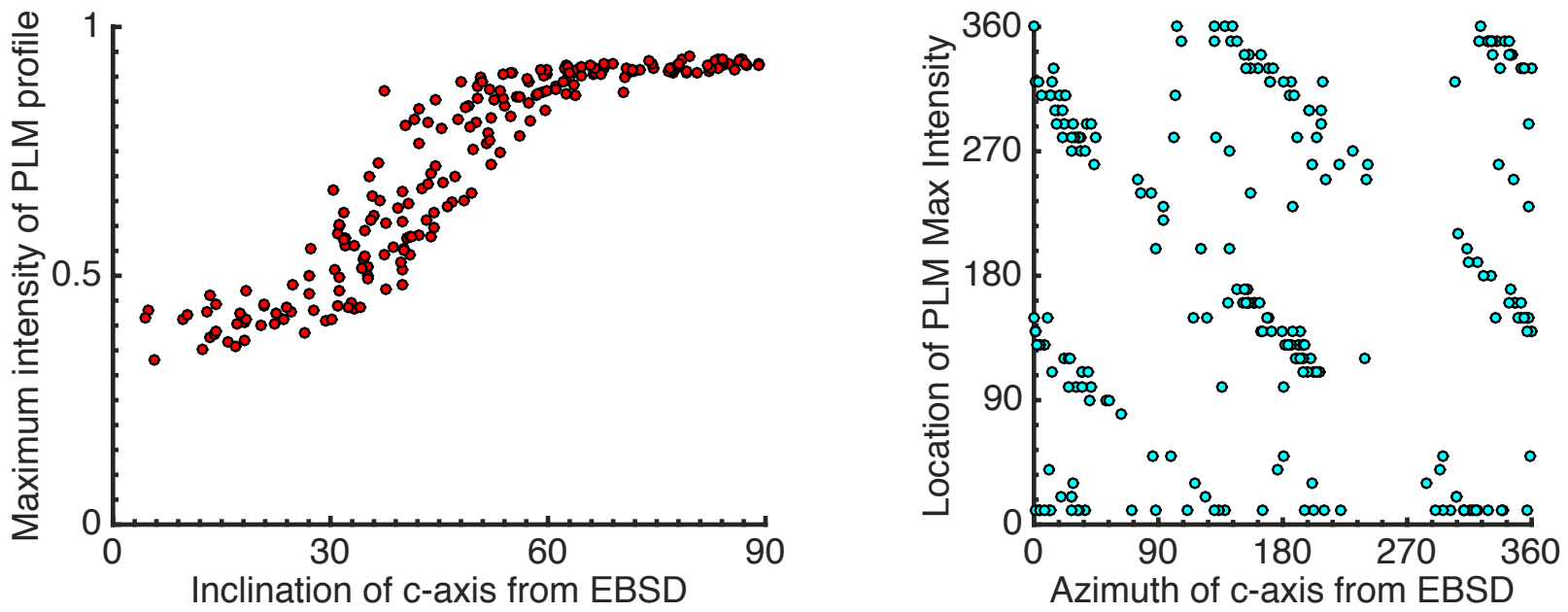

Figure 2. Left: Maximum intensity recorded from polarized light intensity profiles vs. degrees inclination of $c$-axis calculated from EBSD data. Right: Sample rotation at maximum intensity extracted from the polarized light intensity profile vs. azimuthal angle derived from EBSD. 